\title{
BMJ Open Developing and validating utility parameters to establish patient-reported outcome-based perioperative symptom management in patients with lung cancer: a multicentre, prospective, observational cohort study protocol
}

To cite: Dai W, Xie S, Zhang R, et al. Developing and validating utility parameters to establish patient-reported outcomebased perioperative symptom management in patients with lung cancer: a multicentre, prospective, observational cohort study protocol. BMJ Open 2019;9:e030726. doi:10.1136/ bmjopen-2019-030726

- Prepublication history for this paper is available online. To view these files, please visit the journal online (http://dx.doi. org/10.1136/bmjopen-2019030726).

WD and SX contributed equally.

Received 28 March 2019 Revised 27 September 2019 Accepted 07 October 2019

\section{Check for updates}

(c) Author(s) (or their employer(s)) 2019. Re-use permitted under CC BY-NC. No commercial re-use. See rights and permissions. Published by BMJ.

For numbered affiliations see end of article.

Correspondence to Dr Qiuling Shi; qshi@mdanderson.org

Dr Xiaojun Yang; xwksch@163.com

\section{ABSTRACT}

Introduction Patient-reported outcome-based symptom monitoring and alerting have been attractive for patient care after a tumour-removal surgery. However, the implementation parameters of this patient-centred symptom management system in perioperative patients with lung cancer are still lacking. We aim to develop a perioperative symptom scale (PSS) for monitoring, to determine the optimal time points for symptom assessment and to define the alert thresholds for medical intervention.

Methods and analysis This study will prospectively recruit 300 patients undergoing lung cancer surgery in six hospitals. The MD Anderson Symptom Inventory-Lung Cancer Module (MDASI-LC) is used to collect longitudinal symptom data preoperatively, daily postoperatively during in-hospital stay and weekly after discharge until 4 weeks or the start of postoperative oncological therapy. Symptoms that change significantly over time will be generated as the PSS. We will determine the optimal time points for follow-up using the generalised linear mixedeffects models. The MDASI-LC interference-measured functional status will be used as the anchor for the alert thresholds.

Ethics and dissemination Ethics Committee of Sichuan Cancer Hospital approved this study on 16 October 2017 (No. SCCHEC-02-2017-042). The manuscript is based on the latest protocol of Version 3.0, 15 September 2019. The results of this study will be presented at medical conferences and published in peer-reviewed journals. Trials registration number NCT03341377.

\section{INTRODUCTION}

Lung cancer is the leading cause of death among all types of cancers, ${ }^{12}$ with surgery as one of its main treatment methods. In 2015, 147000 lung cancer surgeries were performed in tertiary hospitals in China. ${ }^{3}$ Thoracotomy or minimally invasive

\section{Strengths and limitations of this study}

- This is a multicentre, prospective, observational cohort study from the real-world clinical setting in China.

- It focuses on developing and validating utility parameters for future implementation of patient-reported outcome-based perioperative symptom management in patients with lung cancer.

- It focuses on frequent perioperative longitudinal symptom data collection, including preoperatively, daily postoperatively during in-hospital stay and weekly after discharge until 4 weeks or the start of postoperative oncological therapy.

- The MD Anderson Symptom Inventory-Lung Cancer Module is used to collect symptom data.

- The fact that five subcentres joined the study midway may be a limitation.

thoracoscopic surgery can lead to severe and various postoperative symptoms, such as pain, fatigue, cough and shortness of breath. ${ }^{4-9}$ Adequate perioperative symptom control can accelerate postoperative recovery, improve quality of life (QOL) and ensure timely return to intended oncological therapy, and thus, potentially benefit survival. ${ }^{10} 11$ Clinical trials have shown that the use of patient-reported outcome (PRO)-based symptom monitoring in patients receiving chemotherapy can not only improve QOL but also significantly improve survival. ${ }^{12-14}$ However, very few studies have been conducted in the perioperative patients with lung cancer. ${ }^{1015}$

PRO-based symptom management is the key and ideal model for patient-centred care. ${ }^{15-19}$ However, there are still a few 
technical and methodological issues to be resolved before implementing PRO tools in perioperative symptom management in patients with lung cancer. First, a brief lung cancer surgery-specific measurement scale is lacking. Currently, four commonly used lung cancer-specific PRO tools are available: the European Organisation for Research and Treatment of Cancer Quality of Life Questionnaire-Lung Cancer Module, the Functional Assessment of Cancer Therapy-Lung, the Lung Cancer Symptom Scale and the MD Anderson Symptom Inventory-Lung Cancer Module (MDASI-LC). ${ }^{16}{ }^{20-24}$ These scales are primarily generated and validated in patients with lung cancer receiving chemotherapy and radiotherapy. It is still unknown whether these items will be appropriate for patients undergoing lung cancer surgery. More importantly, there are too many items on these instruments that hinder clinical application. Second, the key symptom monitoring time points are undetermined. Usually, PRO data collection for discharged patients who have undergone lung cancer surgery is limited to follow-up clinic visits. The first follow-up clinic visit is $\sim 4$ weeks after discharge. During these 4 weeks, the patient's symptoms and functional status can change rapidly, ${ }^{18}$ and these potential abnormalities are often ignored, leading to negative clinical outcomes, for instance, postoperative complications, unplanned clinic visit or emergency room visit. In addition, the absence of key PRO information influences the evaluation of clinical outcomes. ${ }^{25}$ Third, evidence-based alert thresholds for perioperative intervention are lacking. A definitive cut-off point of symptom score is the premise of patient symptom monitoring and precision medical intervention. In this study, we aim to solve these methodological issues, via developing and validating a perioperative symptom scale (PSS) for symptom monitoring, determining the optimal time points for symptom assessment and defining the alert thresholds for medical intervention.

\section{METHODS AND ANALYSIS \\ Study design}

This is a real-world, ongoing, multicentre, prospective, observational cohort study. A flow diagram of this study is shown in figure 1.

\section{Setting}

The study is being conducted in six hospitals in China, namely, Sichuan Cancer Hospital, The Third People's Hospital of Chengdu, The Seventh People's Hospital of Chengdu, Jiangyou People's Hospital, Zigong First People's Hospital and Dazhu County People's Hospital. This study was initiated by Sichuan Cancer Hospital and started on November 2017. The other five research centres joined the study in January 2019. This study is estimated to be completed before 31 March 2020.

\section{Study population}

Eligible patients are required to be aged $\geq 18$ years, have no cognitive impairment or be able to understand the

\section{Enrolment}

Eligibility assessment

\section{Data Collection (in-hospital)}

Clinical characteristics, patient-reported outcomes, et al Preoperatively and daily postoperatively

\section{Follow-up (after discharge)}

Patient-reported outcomes, follow-up information, et al Weekly until 4 weeks or the start of postoperative cancer therapy

\section{Data Analysis}

Perioperative symptom scale, time point, alert threshold

Figure 1 Flow diagram of this study.

study requirements, be pathologically or clinically diagnosed as primary lung cancer before surgery, and plan to undergo a surgical procedure.

\section{Sample size calculation}

This study primarily aims to establish a symptom scale that can be used to monitor perioperative symptom burden in patients with lung cancer and that requires significant changes over time during the perioperative period (from preoperatively to 4 weeks after discharge). Our preliminary work showed that the overall SD of the score of the main symptom of lung cancer (0-10 score) was 2.2 in the first postoperative month. With an average of 5 assessments, the rate of symptom score change over time was 0.1 points per assessment according to the general linear regression model. In order to reject the null hypothesis that the symptom scale does not change significantly over time, 239 patients with effective symptom data are needed. The type I error rate is $5 \%$, and the power is $80 \%$. In consideration of $20 \%$ attrition, the final sample size is $\sim 300(239 / 0.8)$ cases.

\section{Outcome measures}

Primary outcome in this study is perioperative symptom burden in patients with lung cancer, as measured by the MDASI-LC. The symptom burden will be presented via a PSS, generated from a subgroup of MDASI-LC symptom items that change significantly over the perioperative period. The MDASI-LC is used to collect longitudinal symptom data preoperatively (typically within 3 days before surgery), daily postoperatively (in-hospital stay of $\leq 14$ days) and weekly after discharge until 4 weeks $( \pm 3$ days) or the start of postoperative cancer therapy. The MDASI-LC is a lung cancer-specific PRO measurement, which has been translated and validated in a Chinese 
setting. Secondary outcomes mainly include QOL measured by a single-item QOL scale (UNISCALE) ${ }^{26}$ and functional status measured by MDASI-LC interference items. The measurement time points of QOL are the same as that of the MDASI-LC. We also measure patient's perception of symptom and daily functioning changes via a five-point Likert Scale weekly after discharge until 4 weeks ( \pm 3 days) or the start of postoperative cancer therapy.

\section{Withdrawal criteria}

Participants will be withdrawn from this study if they meet the following criteria: (1) cancellation of planned surgery, (2) >24hours of postoperative endotracheal intubation in the Intensive Care Unit, (3) postoperative length of hospital stay of $>14$ days, (4) severe complications interfering with PRO data collection, (5) postoperative pathological diagnosis is not primary lung cancer, (6) those who do not follow the study protocol (deliberately providing incorrect PRO data), (7) those who ask to withdraw from the research or (8) other conditions that require withdrawal as assessed by the investigator.

\section{Data collection, management and monitoring}

WeuseREDCap, ${ }^{2728}$ aweb-basedsoftwareapplicationfordata storage and management (http://125.71.214.100:888/ redcap), to store and manage data. Electronic case report form was designed on REDCap. It consists of 12 data collection instruments, namely, demographic characteristics, preoperative characteristics, surgery information, anaesthesia information, postoperative care, perioperative complications, pain management, MDASI-LC, QOL, symptom and daily functioning changes, completion data and follow-up information. PRO data are collected using a paper questionnaire or an e-questionnaire and then recorded in REDCap. Participants are instructed to fill out the scales independently. If they have difficulties in completing the scales, investigators or other proxies will assist them by reading each item aloud and recording their responses. All data are deidentified and entered into the REDCap platform. Data are entered by a data entry clerk and checked regularly by a quality controller. Data monitoring is carried out regularly by the Ethics Committee of Sichuan Cancer Hospital.

\section{Quality control}

Investigators received standard operating procedure training before recruiting the patients. The subcentres receive regular online directions, telephone monitoring and on-site supervision conducted by the principal investigator or international expert from MD Anderson Cancer Center.

\section{Data analysis}

For inclusion in the final analysis, the participants must complete the MDASI-LC assessments preoperatively and at least two additional assessments postoperatively. The multiple imputation method will be used to impute missing data. Continuous data will be expressed as mean \pm SD or median and IQR. Categorical data will be presented as number and percentage. We will use generalised linear mixed-effects models to describe trajectories of symptom severity, symptom interference and QOL during the entire investigation period. Considering the time variable, days from surgery, as a continuous variable, symptoms that change significantly over time will be generated as the targets for perioperative symptom monitoring. The PSS score will be obtained by averaging scores of all targeted symptoms. Treating days from surgery as a categorical variable, we will estimate the change of PSS from the previous assessment using a generalised linear mixed-effects model. The optimal time points for monitoring will be determined as those with significant changes in PSS. The alert thresholds will be generated as cut-off points for the PSS with the method proposed by Serlin et $a l,{ }^{29}$ using the 6 MDASI-LC interference items as the anchor. The 6 MDASI interference items have been validated as a reliable and sensitive measure for functional status of patients undergoing cancer treatment ${ }^{1930}$ and 'functional recovery has been considered as the most important target' for postoperative recovery by a large group of international professionals. ${ }^{31}$ Differences are considered statistically significant if the two-tailed $p$ values of $<0.05$. All data analyses will be performed using the SAS V.9.4.

\section{Patient and public involvement}

Patients and the general public were not involved in the design, recruitment and implementation of the study. We have no plans of informing the study participants regarding the results of this study. However, the results will be disseminated to the applicants in the form of a published article as requested.

\section{ETHICS AND DISSEMINATION}

Any amendments to the research protocol will be submitted for ethical approval. All participants must provide informed consent. The results in this study will be first reported at relevant medical conferences and then will eventually be published in peer-reviewed journals.

\section{Author affiliations}

${ }^{1}$ Department of Thoracic Surgery, Sichuan Cancer Hospital and Institute, Sichuan Cancer Center, School of Medicine, University of Electronic Science and Technology of China, Chengdu, China

${ }^{2}$ Graduate School, Chengdu Medical College, Chengdu, China

${ }^{3}$ Department of Thoracic Surgery, The Seventh People's Hospital of Chengdu,

Chengdu, China

${ }^{4}$ Department of Cardiothoracic Surgery, Zigong First People's Hospital, Zigong, China ${ }^{5}$ Department of Thoracic and Cardiovascular Surgery, Jiangyou People's Hospital, Jiangyou, China

${ }^{6}$ Department of Cardiothoracic Surgical Oncology, Dazhu County People's Hospital, Dazhu County, China

${ }^{7}$ Department of Thoracic Surgery, The Third People's Hospital of Chengdu, Chengdu, China

${ }^{8}$ Department of Symptom Research, The University of Texas MD Anderson Cancer Center, Houston, Texas, USA

Acknowledgements We thank all the participants and their advisors involving in this study. We thank Ping Gui and Yaqian Feng for their data entry work. 
Contributors WD, YJ, JH, QL, XY and QS contributed to the study design. WD, SX, RZ, XW, CW, YZ, WF, XL, YM, HZ and XC performed the study. WD, SX, RZ, XW and $C W$ drafted the initial manuscript. $Q L, X Y$ and $Q S$ revised the draft. All authors have reviewed and approved the final manuscript.

Funding This work was supported by Sichuan Science and Technology Program (grant number: 2011FZ0069 and 2019YFH0070), and National Natural Science Foundation of China (grant number: 81872506).

Competing interests None declared.

Patient consent for publication Not required.

Ethics approval This study was approved by the Ethics Committee of Sichuan Cancer Hospital on 16 October 2017 (No. SCCHEC-02-2017-042).

Provenance and peer review Not commissioned; externally peer reviewed.

Open access This is an open access article distributed in accordance with the Creative Commons Attribution Non Commercial (CC BY-NC 4.0) license, which permits others to distribute, remix, adapt, build upon this work non-commercially, and license their derivative works on different terms, provided the original work is properly cited, appropriate credit is given, any changes made indicated, and the use is non-commercial. See: http://creativecommons.org/licenses/by-nc/4.0/.

ORCID iD

Wei Dai http://orcid.org/0000-0003-1650-1590

\section{REFERENCES}

1 Siegel RL, Miller KD, Jemal A. Cancer statistics, 2018. CA Cancer J Clin 2018;68:7-30.

2 Bray F, Ferlay J, Soerjomataram I, et al. Global cancer statistics 2018: GLOBOCAN estimates of incidence and mortality worldwide for 36 cancers in 185 countries. CA Cancer J Clin 2018;68:394-424.

3 Liao H, Mei JD, Liu CW, et al. [A survey on the current development of thoracic surgery in tertiary hospitals of China]. Zhonghua Wai Ke Za Zhi 2018;56:888-91.

4 Lowery AE, Krebs P, Coups EJ, et al. Impact of symptom burden in post-surgical non-small cell lung cancer survivors. Support Care Cancer 2014;22:173-80.

5 Oksholm T, Rustoen T, Cooper B, et al. Trajectories of symptom occurrence and severity from before through five months after lung cancer surgery. J Pain Symptom Manage 2015;49:995-1015.

6 Yang P, Cheville AL, Wampfler JA, et al. Quality of life and symptom burden among long-term lung cancer survivors. J Thorac Oncol 2012;7:64-70.

7 Kenny PM, King MT, Viney RC, et al. Quality of life and survival in the 2 years after surgery for non small-cell lung cancer. $J$ Clin Oncol 2008;26:233-41.

8 Li WW, Lee TW, Lam SS, et al. Quality of life following lung cancer resection: video-assisted thoracic surgery vs thoracotomy. Chest 2002:122:584-9.

9 Bendixen M, Jørgensen OD, Kronborg C, et al. Postoperative pain and quality of life after lobectomy via video-assisted thoracoscopic surgery or anterolateral thoracotomy for early stage lung cancer: a randomised controlled trial. Lancet Oncol 2016;17:836-44.

10 Cleeland CS, Wang XS, Shi Q, et al. Automated symptom alerts reduce postoperative symptom severity after cancer surgery: a randomized controlled clinical trial. J Clin Oncol 2011;29:994-1000.

11 Miralpeix E, Nick AM, Meyer LA, et al. A call for new standard of care in perioperative gynecologic oncology practice: impact of enhanced recovery after surgery (ERAS) programs. Gynecol Oncol 2016:141:371-8.

12 Basch E, Deal AM, Kris MG, et al. Symptom monitoring with patientreported outcomes during routine cancer treatment: a randomized controlled trial. J Clin Oncol 2016;34:557-65.

13 Basch E, Deal AM, Dueck AC, et al. Overall survival results of a trial assessing patient-reported outcomes for symptom monitoring during routine cancer treatment. JAMA 2017;318:197-8.

14 Denis F, Basch E, Septans AL, et al. Two-year survival comparing web-based symptom monitoring vs routine surveillance following treatment for lung cancer. JAMA 2019;321:306-7.

15 Khullar OV, Fernandez FG. Patient-reported outcomes in thoracic surgery. Thorac Surg Clin 2017;27:279-90.

16 Bouazza YB, Chiairi I, El Kharbouchi O, et al. Patient-reported outcome measures (PROMs) in the management of lung cancer: a systematic review. Lung Cancer 2017;113:140-51.

17 Basch E. Patient-reported outcomes - harnessing patients' voices to improve clinical care. N Engl J Med 2017;376:105-8.

18 Fagundes CP, Shi Q, Vaporciyan AA, et al. Symptom recovery after thoracic surgery: measuring patient-reported outcomes with the MD Anderson symptom inventory. J Thorac Cardiovasc Surg 2015;150:613-9.e2.

19 Shi Q, Wang XS, Vaporciyan AA, et al. Patient-reported symptom interference as a measure of postsurgery functional recovery in lung cancer. J Pain Symptom Manage 2016;52:822-31.

20 Damm K, Roeske N, Jacob C. Health-related quality of life questionnaires in lung cancer trials: a systematic literature review. Health Econ Rev 2013;3:15.

21 Bergman B, Aaronson NK, Ahmedzai S, et al. The EORTC QLQLC13: a modular supplement to the EORTC core quality of life questionnaire (QLQ-C30) for use in lung cancer clinical trials. EORTC Study Group on quality of life. Eur J Cancer 1994;30A:635-42.

22 Cella D. The functional assessment of cancer therapy-lung and lung cancer subscale assess quality of life and meaningful symptom improvement in lung cancer. Semin Oncol 2004;31:11-5.

23 Hollen PJ, Gralla RJ, Kris MG, et al. Measurement of quality of life in patients with lung cancer in multicenter trials of new therapies. Psychometric assessment of the lung cancer symptom scale. Cancer 1994;73:2087-98.

24 Mendoza TR, Wang XS, Lu C, et al. Measuring the symptom burden of lung cancer: the validity and utility of the lung cancer module of the M. D. Anderson symptom inventory. Oncologist 2011;16:217-27.

25 Calvert M, Kyte D, Duffy H, et al. Patient-reported outcome (PRO) assessment in clinical trials: a systematic review of guidance for trial protocol writers. PLoS One 2014;9:e110216.

26 Sloan JA, Loprinzi CL, Kuross SA, et al. Randomized comparison of four tools measuring overall quality of life in patients with advanced cancer. J Clin Oncol 1998;16:3662-73.

27 Harvey LA. REDCap: web-based software for all types of data storage and collection. Spinal Cord 2018;56:625.

28 Klipin M, Mare I, Hazelhurst S, et al. The process of installing REDCap, a web based database supporting biomedical research: the first year. App/ Clin Inform 2014;5:916-29.

29 Serlin RC, Mendoza TR, Nakamura Y, et al. When is cancer pain mild, moderate or severe? grading pain severity by its interference with function. Pain 1995;61:277-84.

30 Shi Q, Mendoza TR, Wang XS, et al. Using a symptom-specific instrument to measure patient-reported daily functioning in patients with cancer. Eur J Cancer 2016;67:83-90.

31 Aahlin EK, von Meyenfeldt $\mathrm{M}$, Dejong $\mathrm{CH}$, et al. Functional recovery is considered the most important target: a survey of dedicated professionals. Perioper Med (Lond) 2014;3:5. 